\title{
Kaon decays at KLOE-2
}

\author{
Giancarlo D'Ambrosio ${ }^{1, \star}$ \\ ${ }^{1}$ INFN-Sezione di Napoli, Via Cintia, 80126 Napoli, Italia
}

\begin{abstract}
I review kaon decays. I introduce the flavor problem and possible solutions. Very rare kaon decays like $K \rightarrow \pi v \bar{v}$ are very important to this purpose: we study also $K \rightarrow \pi l^{+} l^{-}, K \rightarrow \pi \pi e e$ where chiral dynamics is important to disentangle short distance effects. We have also studied lepton flavor (universality) violation in rare kaon decays and the Bardeen Buras Gerard approach to describe the $K^{ \pm} \rightarrow \pi^{ \pm} l^{+} l^{-}$form factor.
\end{abstract}

\section{Introduction}

Kaon decays are an important place to study non-perturbative aspects of QCD and test the Standard Model. Indeed some channels are completely dominated by long-distance dynamics, such as the CPconserving amplitude for $K \rightarrow \pi \pi$ and others, like $K \rightarrow \pi v \bar{v}$ [1], which are described in terms of pure short-distance physics. In this review we will be mostly concerned with kaon decays involving electromagnetic interactions and thus long-distance phenomena are not negligible. However, as we shall see, it is still possible in these channels to extract the short-distance component with an accurate analysis. Indeed there are plenty of motivations to look for new physics (NP) in these kaon decays [2].

The channels which will be considered here are $K \rightarrow \pi \pi \gamma, K \rightarrow \pi \pi e e, K_{S, L} \rightarrow l^{+} l^{-}, K_{S, L} \rightarrow$ $l^{+} l^{-} l^{+} l^{-}$and $K_{S} \rightarrow \pi^{+} \pi^{-} l^{+} l^{-}$. Recently, Buras and collaborators, motivated by their previous work where weak hadronic matrix elements are evaluated in their model (BBG model) and also by recent lattice results, are investigating New Physics to explain the experimental value of $\epsilon^{\prime}$ [2-5], Motivated by this interest we study also the $K^{ \pm} \rightarrow \pi^{ \pm} l^{+} l^{-}$form factor in the BBG model [6].

\section{THE ULTRA-RARE DECAY $K^{+} \rightarrow \pi^{+} v \bar{v}$}

Rare kaon decays furnish challenging MFV probes and will severely constrain additional flavor physics motivated by NP. SM predicts the $V-A \otimes V-A$ effective hamiltonian

$$
\mathcal{H}=\frac{G_{F}}{\sqrt{2}} \frac{\alpha}{2 \pi \sin ^{2} \theta_{W}}(\underbrace{V_{c s}^{*} V_{c d} X_{N L}}_{\lambda x_{c}}+\underbrace{V_{t s}^{*} V_{t d} X\left(x_{t}\right)}_{A^{2} \lambda^{5}(1-\rho-i \eta) x_{t}}) \bar{s}_{L} \gamma_{\mu} d_{L} \bar{v}_{L} \gamma^{\mu} v_{L},
$$

$x_{q}=m_{q}^{2} / M_{W}^{2}, \theta_{W}$ the Weak angle and $X$ 's are the Inami-Lin functions with Wilson coefficients known at two-loop electroweak corrections [7]. $S U(2)$ isospin symmetry relates hadronic matrix elements

\footnotetext{
^e-mail: gdambros@na.infn.it
} 
for $K \rightarrow \pi v \bar{v}$ to $K \rightarrow \pi l \bar{v}$ to a very good precision [8] while long distance contributions and QCD corrections are under control [7] and the main uncertainties is due to the strong corrections in the charm loop contribution. The structure in (1) leads to a pure CP violating contribution to $K_{L} \rightarrow \pi^{0} v \bar{v}$, induced only from the top loop contribution and thus proportional to $\mathfrak{J} m\left(\lambda_{t}\right)\left(\lambda_{t}=V_{t s}^{*} V_{t d}\right)$ and free of hadronic uncertainties. This leads to the prediction [7]

$$
\begin{array}{lc}
\mathcal{B}\left(K_{L} \rightarrow \pi^{0} v \bar{v}\right)_{\mathrm{SM}}= & (2.9 \pm 0.2 \pm 0.0) \times 10^{-11} \\
\mathcal{B}\left(K^{+} \rightarrow \pi^{+} v \bar{v}\right)_{\mathrm{SM}}= & (8.3 \pm 0.3 \pm 0.3) \times 10^{-11}
\end{array}
$$

where the first is the parametric uncertainty due to the error on $\left|V_{c b}\right|, \rho$ and $\eta, f_{K}$, and the second error summarizes the theoretical uncertainties on non-perturbative physics and QCD higher order terms.

$K^{ \pm} \rightarrow \pi^{ \pm} v \bar{v}$ receives CP conserving contributions proportional to $\mathfrak{R} e\left(\lambda_{c}\right)$, and to $\mathfrak{R} e\left(\lambda_{t}\right)$ and a CP violating one proportional to $\mathfrak{J} m\left(\lambda_{t}\right)$. E949 Collaboration [9, 10] and E391a Collaboration [11] have then measured

$$
\begin{gathered}
\mathcal{B}\left(K^{ \pm}\right)=\left(1.73_{-1.05}^{+1.15}\right) \times 10^{-10} \quad \text { E949 } \\
\mathcal{B}\left(K_{L}\right)<2.6 \times 10^{-8} \text { at } 90 \% \text { C.L. } \quad \text { E391aCollaboration }
\end{gathered}
$$

The direct upper bound for the neutral decay can be improved with a theoretical analysis: the isospin structure of any $\bar{s} d$ operator (bilinear in the quark fields) leads to the model independent relation among $A\left(K_{L} \rightarrow \pi^{0} v \bar{v}\right)$ and $A\left(K^{ \pm} \rightarrow \pi^{ \pm} v \bar{v}\right)$ [12]; this leads to

$$
\mathcal{B}\left(K_{L} \rightarrow \pi^{0} v \bar{v}\right)<4 \mathcal{B}\left(K^{ \pm} \rightarrow \pi^{ \pm} v \bar{v}\right)
$$

The upcoming KOTO experiment $[8,13]$ for $K_{L} \rightarrow \pi^{0} v \bar{v}$ and NA62 at CERN [14] encourage theoretical investigations of extensions of the SM: these experiments probe deeply to the MFV scale [15]. More aggressive NP models can furnish substantial enhancements and be either discovered or ruled out $[7,16]$ ! Further motivation to rare kaon decays program has come from the possible departure from the SM prediction of the experimental value of $\epsilon^{\prime}[2-5,9]$ : we have investigated a very predictive Susy scenario for $K \rightarrow \pi v \bar{v}[17,18]$

\section{$3 \quad K \rightarrow \pi \pi \gamma$ and $K \rightarrow \pi \pi e e$-decays}

\section{1 $K \rightarrow \pi \pi \gamma$}

Let's discuss $K(p) \rightarrow \pi\left(p_{1}\right) \pi\left(p_{2}\right) \gamma(q)$ decays: according to gauge and Lorentz invariance we decompose $K(p) \rightarrow \pi\left(p_{1}\right) \pi\left(p_{2}\right) \gamma(q)$ decays, in electric $(E)$ and magnetic $(M)$ amplitudes [19]. Particularly interesting are the recent interesting NA48/2 data regarding $K^{+} \rightarrow \pi^{+} \pi^{0} \gamma$ decays [20].

Due to the $\Delta I=3 / 2$ suppression of the bremsstrahlung, interference between $E_{B}$ and the electric dipole $\left(E_{D E}\right)$ and magnetic transitions $\left(M_{D E}\right)$ can be measured. Defining $z_{i}=p_{i} \cdot q / m_{K}^{2} \quad z_{3}=$ $p_{K} \cdot q / m_{K}^{2}$ and $z_{3} z_{+}=\frac{m_{\pi^{+}}^{2}}{m_{K}^{2}} W^{2},\left(W^{2}\right.$ is defined in this equation) we can study the deviation from bremsstrahlung

$$
\frac{\partial^{2} \Gamma}{\partial T_{c}^{*} \partial W^{2}}=\frac{\partial^{2} \Gamma_{I B}}{\partial T_{c}^{*} \partial W^{2}}\left[1+\frac{m_{\pi^{+}}^{2}}{m_{K}} 2 \operatorname{Re}\left(\frac{E_{D E}}{e A}\right) W^{2}+\frac{m_{\pi^{+}}^{4}}{m_{K}^{2}}\left(\left|\frac{E_{D E}}{e A}\right|^{2}+\left|\frac{M_{D E}}{e A}\right|^{2}\right) W^{4}\right]
$$

where $A=A\left(K^{+} \rightarrow \pi^{+} \pi^{0}\right)$. Study of the Dalitz plot relative to Eq. (6) has lead to measure separately the linear and the quadratic term in $W^{2}$ : indeed integrating over the Dalitz plot and dividing for the IB 
Branching ratio the right-hand side of Eq. (6) leads these two terms (linear and quadratic) respectively to the NA48/2 measurement of the two fractions, $\operatorname{Frac}(I N T)$ and $\operatorname{Frac}(D E)$, (see Ref. [20] )

\begin{tabular}{|c|c|}
\hline \multicolumn{2}{|c|}{ NA48/2 $K^{+} \rightarrow \pi^{+} \pi^{0} \gamma$ results } \\
\hline NA48/2 & $T_{c}^{*} \in[0,80] \mathrm{MeV}$ \\
\hline $\operatorname{Frac}(D E)=$ & $(3.32 \pm 0.15 \pm 0.14) \times 10^{-2}$ \\
\hline $\operatorname{Frac}(I N T)=$ & $(-2.35 \pm 0.35 \pm 0.39) \times 10^{-2}$ \\
\hline
\end{tabular}

\section{2 $K \rightarrow \pi \pi e e$-decays}

Historically kaon four body semileptonic decays, $K_{e 4}$ have been studied as a tool to tackle final state rescattering effects in $K \rightarrow \pi \pi$-decays: crucial to this goal has been finding an appropriate set of kinematical variables which would allow i) to treat the system as two body decay in dipion mass $M_{\pi \pi}$ and dilepton mass $\mathrm{M}_{l^{+} l^{-}}$and ii) to identify appropriate kinematical asymmetries to extract observables crucially dependent on final state interaction [21].

The traditional kinematical variables for the four body kaon semileptonic decay allow to write the four body phase space $\Phi$ in terms of the two two-body phase spaces: $\Phi_{\pi}$ and $\Phi_{\ell}$ from $[21,22]$. We extend the $K_{e 4}$ kinematical and dynamical description of the amplitude to describe the $K \rightarrow \pi \pi e^{+} e^{-}$ decays; in particular we write the long distance contribution as

$$
\mathcal{M}_{L D}=\frac{e}{q^{2}}\left[\bar{u}\left(k_{-}\right) \gamma^{\mu} v\left(k_{+}\right)\right] H_{\mu}\left(p_{1}, p_{2}, q\right),
$$

where $H_{\mu}$ is the electroweak hadronic vector, which can be written in terms of three form factors $F_{1,2,3}$ :

$$
H^{\mu}\left(p_{1}, p_{2}, q\right)=F_{1} p_{1}^{\mu}+F_{2} p_{2}^{\mu}+F_{3} \varepsilon^{\mu \nu \alpha \beta} p_{1 v} p_{2 \alpha} q_{\beta} .
$$

Then the kinematical distributions can be written in terms of the three form factors.

Short distance physics can be studied by the diplane angular asymmetry. However this observable is proportional to electric (bremsstrahlung) and magnetic interference, both contributions known already from $K_{L} \rightarrow \pi^{+} \pi^{-} \gamma$. In fact it was known that these contributions were large and they may obscure smaller but more interesting short distance physics effects.

We have performed a similar analysis for the decay $K^{+} \rightarrow \pi^{+} \pi^{0} e^{+} e^{-}$trying to focus on i) short distance physics and ii) all possible Dalitz plot analyses to disentangle all possible interesting long and short distance effects [22]. The interesting physics is hidden by bremsstrahlung [22-24]

$$
\begin{array}{cc}
\mathcal{B}\left(K^{+} \rightarrow \pi^{+} \pi^{0} e^{+} e^{-}\right)_{B} & \sim(330 \pm 15) \cdot 10^{-8} \\
\mathcal{B}\left(K^{+} \rightarrow \pi^{+} \pi^{0} e^{+} e^{-}\right)_{M} & \sim(6.14 \pm 1.30) \cdot 10^{-8},
\end{array}
$$

and so Dalitz plot analysis is necessary in order to capture the more interesting direct emission contributions. The $K^{+} \rightarrow \pi^{+} \pi^{0} e^{+} e^{-}$-amplitude is then written as in Eq. 7 optimizing the theoretical and experimental knowledge of $K(p) \rightarrow \pi\left(p_{1}\right) \pi\left(p_{2}\right) \gamma(q)$ decays.

Relatively to the $K^{ \pm} \rightarrow \pi^{ \pm} \pi^{0} \gamma$ the possibility to go kinematically at large $q^{2}$ opens the possibility to beat the bremsstrahlung: at large dilepton invariant mass the bremsstrahlung can be even 100 times smaller than the magnetic contribution, at the price, however, of decreasing the statistics. Indeed compared to $K^{ \pm} \rightarrow \pi^{ \pm} \pi^{0} \gamma$ differential decay rates we have the possibility to measure also the $q^{2}$ differential rates

$$
\frac{\partial^{2} \Gamma_{I B}}{\partial T_{c}^{*} \partial W^{2} \partial q^{2}}
$$


The further advantage is that at each $q^{2}$ we can measure the different Dalitz plot. Also several short distance observables can be measured by appropriate kinematical analyses [22, 23]. In Table 1 we show the ratios of Bremsstrahlung (B) to magnetic (M) and to various interferences. Our effort has been to write the $K^{ \pm} \rightarrow \pi^{ \pm} \pi^{0} e^{+} e^{-}$amplitude in terms of experimental known Bremsstrahlung (B) and $\mathrm{DE}$, electric (E) and magnetic (M), transitions [22]

Table 1. Branching ratios for the Bremsstrahlung and the relative weight of the rest of the contributions for different cuts in $q$, starting at $q_{\min }$ (first row) and ending at $180 \mathrm{MeV}$. In the last column we have also included the parity-odd magnetic-electric interference term.

\begin{tabular}{llllll}
\hline$q_{c}(\mathrm{MeV})$ & $\mathrm{B}\left[10^{-8}\right]$ & $\mathrm{B} / \mathrm{M}$ & $\mathrm{B} / \mathrm{E}$ & $\mathrm{B} / \mathrm{BE}$ & $\mathrm{B} / \mathrm{BM}$ \\
\hline $2 m_{l}$ & 418.27 & 71 & 4405 & 128 & 208 \\
55 & 5.62 & 12 & 118 & 38 & 44 \\
100 & 0.67 & 8 & 30 & 71 & 36 \\
180 & 0.003 & 12 & 5 & -19 & 44 \\
\hline
\end{tabular}

The big news is that NA48/2 has reported a final measurement of this branching: the experiment selects 3 reconstructed tracks coming from one decay vertex, then Particle ID for $e^{ \pm} / \pi^{ \pm}$separation; then two reconstructed $\gamma$ clusters are compatible with $\pi^{0}$ mass [25]. The number of $K^{ \pm}$decays (kaon flux) is measured by using the reference channel $K^{ \pm} \rightarrow \pi^{ \pm} \pi^{0}(\gamma)$. 5076 events have been selected with 289 background; the error is dominated by external error on $\mathcal{B}\left(\pi^{0} \rightarrow e^{+} e^{-} \gamma\right)$

$$
\left(4.06 \pm 0.06_{\text {stat. }} \pm 0.04_{\text {syst. }} \pm 0.13_{\text {ext. }}\right) \times 10^{-6}
$$

to be compared to our prediction dominated from Bremsstrahlung [22, 23]

$$
\begin{array}{ccc}
\mathcal{B}(I B)=(4.19) \times 10^{-6} & \text { no Isospin breaking } & \mathcal{B}(\text { TOT })=(4.29) \times 10^{-6} \\
\mathcal{B}(I B)=(4.10) \times 10^{-6} & \text { with Isospin breaking } & \mathcal{B}(T O T)=(4.19) \times 10^{-6}
\end{array}
$$

\section{Bardeen Buras Gerard approach and $K^{ \pm} \rightarrow \pi^{ \pm} l^{+} l^{-}$form factor}

Attempting to match long distance to short distance Buras and collaborators [3, 4] compute loop calculations employing a cut-off regulator instead of the dimensional regularization method. Consequently, their results exhibit a quadratic dependence on the physical cut-off $M$ which is lost in the usual chiral perturbative calculations. This quadratic dependence is a crucial ingredient in the matching of the meson and quark pictures. They argue that one can obtain a parametrization of non-perturbative QCD effects by matching a low-energy Lagrangian valid up to the scale $M$, to the logarithmic behavior of relevant Wilson coefficients at high-energy. In this work we refer to this computational method as the Bardeen-Buras-Gérard framework (BBG). Recentely Buras has advocated this determination to claim New Physics to explain the experimental value of $\epsilon^{\prime}$ [5]. BBG evaluate $K \rightarrow \pi \pi$ chiral loop with a dimensional cut-off $(\mathrm{M})$ and match the quadratic divergences of the $Q_{i}(M)$ weak matrix elements with the $\log$ of the short distance Wilson coefficient as described by the equation

$$
H_{\mathrm{eff}}=\sum_{i} c_{i}(\mu) Q_{i}(\mu)
$$

We have studied in the BBG contest the $K^{ \pm} \rightarrow \pi^{ \pm} l^{+} l^{-}$form factor described in CHPT by a loop function, $W_{+}^{\pi \pi}(z)$ and a polynomial in $z$ with experimentally determined coefficients $a_{+}$and $b_{+}$[26]. 


\begin{tabular}{|c|c|c|}
\hline & PDG & Prospects \\
\hline$K_{S} \rightarrow \mu \mu$ & $<9 \times 10^{-9}$ at $90 \% \mathrm{CL}$ & $(\mathrm{LD})(5.0 \pm 1.5) \cdot 10^{-12} \quad \mathrm{NP}<10^{-11}$ \\
\hline$K_{L} \rightarrow \mu \mu$ & $(6.84 \pm 0.11) \times 10^{-9}$ & difficult $:$ SD $<<$ LD \\
\hline$K_{S} \rightarrow \mu \mu \mu \mu$ & - & $\mathrm{SM} L D \sim 2 \times 10^{-14}$ \\
\hline$K_{S} \rightarrow е e \mu \mu$ & - & $\sim 10^{-11}$ \\
\hline$K_{S} \rightarrow$ eeee & - & $\sim 10^{-10}$ \\
\hline$K_{S} \rightarrow \pi^{+} \pi^{-} \mu^{+} \mu^{-}$ & - & $\mathrm{SM} L D \sim 10^{-14}$ \\
\hline
\end{tabular}

Table 2. Interesting channels: PDG values vs theoretical estimates

These parameterize the intermediate region between low and high energy regimes. Our goal is to predict the values of these two coefficients using BBG framework [6].

\section{Lepton flavor (universality) violation in rare kaon decays}

Recent anomalies in the decays of $B$-mesons and the Higgs boson provide hints towards lepton flavor (universality) violating physics beyond the Standard Model. We observe that 4-fermion operators which can explain the $B$-physics anomalies have corresponding analogs in the kaon sector, and we analyze their effect on $K \rightarrow \pi \ell \ell^{\prime}$ and $K \rightarrow \ell \ell^{\prime}$ decays $(\ell=\mu, e)$. For these processes, we point out the corresponding physics opportunities at the NA62 and KOTO experiments. In particular, assuming minimal flavor violation, we comment on the required improvements in sensitivity necessary to test the $B$-physics anomalies in the kaon sector [27].

\section{$6 K_{S, L} \rightarrow l^{+} l^{-}, K_{S, L} \rightarrow l^{+} l^{-} l^{+} l^{-}$and $K_{S} \rightarrow \pi^{+} \pi^{-} l^{+} l^{-}$}

The recent LHCB limit on $K_{S} \rightarrow \mu \bar{\mu}$ [9] in Table 6 is close to test interesting New Physics (NP) models [28]. A high precision measurement can test the short distance (SD) SM but it requires to improve the long distance (LD) prediction [28, 29] with auxiliary channels [30]. $K_{L} \rightarrow \mu \mu$ : the small ratio $\mathrm{SD} / \mathrm{LD} \sim \frac{1}{30}$ may obscure an experimental improvement on the rate [28]. The situation would be a bit ameliorated if the still unknown sign of $A\left(K_{L} \rightarrow \gamma \gamma\right)$ would be either theoretically or experimentally determined. Help to this ambiguity could come from the experimental study of $K_{S . L} \rightarrow l^{+} l^{-} l^{+} l^{-}$[30] As shown in Table 6 these channels are at reach in a high intensity machine and they may also give LD distance info needed for a better control of $K_{L} \rightarrow \mu \mu$. These four body decays have also a peculiar feature, similarly to $K_{S, L} \rightarrow \pi^{+} \pi^{-} e^{+} e^{-}$, the two different helicity amplitudes interfere; then one can measure the sign $K_{L} \rightarrow \rightarrow \gamma^{*} \gamma^{*} \rightarrow l^{+} l^{-} l^{+} l^{-}$by studying the time interference $K_{S} K_{L}$ which it has a decay length $2 \Gamma_{S}[30]$.

Actually only $K^{+} \rightarrow \pi^{+} \pi^{0} e^{+} e^{-}$has been studied so far [22] while $K_{S} \rightarrow \pi^{+} \pi^{-} \mu^{+} \mu^{-}$, more feasible experimentally, is in progress; however generic features can be already extracted from Table I in Ref. [22]: we have less than $10 \mathrm{MeV}$ phase space which can be extracted from the last lines of the Table I in Ref. [22] telling us that i) the Branching is expected $O\left(10^{-14}\right)$ and the novel purely electric and magnetic contribution are relatively enhanced with respect to the less interesting bremsstrahlung.

\section{Conclusions}

In these proceedings we have studied theoretical issues in radiative rare kaon decays. An interesting channel, $K^{+} \rightarrow \pi^{+} \pi^{0} e^{+} e^{-}$, is studied also in connection with the recent experimental NA48 results. 
Motivated by LHCB results on $K_{S} \rightarrow \mu^{+} \mu^{-}$we discuss other channels like $K_{S, L} \rightarrow l^{+} l^{-} l^{+} l^{-}$. Motivated by recent theoretical work by Buras and collaborators we study also the $K^{ \pm} \rightarrow \pi^{ \pm} l^{+} l^{-}$form factor; in this channel may be interesting to study forward-backward asymmetry [26].

\section{Acknowledgements}

I thank the organizers of the workshop, in particular Antonio Di Domenico. Supported in part by MIUR under project 2015P5SBHT and by the INFN research initiative ENP.

\section{References}

[1] V. Cirigliano, G. Ecker, H. Neufeld, A. Pich and J. Portoles, Rev. Mod. Phys. 84, 399 (2012) doi:10.1103/RevModPhys.84.399 [arXiv:1107.6001 [hep-ph]].

[2] A. J. Buras, arXiv:1609.05711 [hep-ph].

[3] W. A. Bardeen, A. J. Buras and J. M. Gerard, Nucl. Phys. B 293 (1987) 787. doi:10.1016/05503213(87)90091-5; A. J. Buras, J. M. Gérard and W. A. Bardeen, "Large N Approach to Kaon Decays and Mixing 28 Years Later: $\Delta I=1 / 2$ Rule, $\hat{B}_{K}$ and $\Delta M_{K}$ ", Eur. Phys. J. C 74, 2871 (2014)

[4] J. M. Gerard, Acta Phys. Polon. B 21 (1990) 257.

[5] A. J. Buras, M. Gorbahn, S. Jager and M. Jamin, JHEP 1511, 202 (2015) doi:10.1007/JHEP11(2015)202 [arXiv:1507.06345 [hep-ph]].

[6] E. Coluccio Leskow, G. D’Ambrosio, D. Greynat and A. Nath, Phys. Rev. D 93, no. 9, 094031 (2016) doi:10.1103/PhysRevD.93.094031 [arXiv:1603.09721 [hep-ph]].

[7] M. Gorbahn, M. Patel and S. Robertson, arXiv:1104.0826 [hep-ph]: J. Brod, M. Gorbahn and E. Stamou, Phys. Rev. D83, 034030 (2011); M. Antonelli, D. M. Asner, D. A. Bauer, T. G. Becher, M. Beneke, A. J. Bevan, M. Blanke and C. Bloise et al., Phys. Rept. 494, 197 (2010) [arXiv:0907.5386 [hep-ph]].

[8] T. K. Komatsubara, Prog. Part. Nucl. Phys. 67, 995 (2012) [arXiv:1203.6437 [hep-ex]].

[9] C. Patrignani et al. [Particle Data Group], Chin. Phys. C 40, no. 10, 100001 (2016). doi:10.1088/1674-1137/40/10/100001

[10] A. V. Artamonov et al. [E949 Collaboration], Phys. Rev. Lett. 101, 191802 (2008) [arXiv:0808.2459 [hep-ex]].

[11] J. K. Ahn et al. [E391a Collaboration], Phys. Rev. D 81, 072004 (2010) [arXiv:0911.4789 [hepex]].

[12] Y. Grossman and Y. Nir, Phys. Lett. B 398, 163 (1997) [hep-ph/9701313].

[13] http://koto.kek.jp/

[14] F. Newson et al., arXiv:1411.0109 [hep-ex]; A. Romano, arXiv:1411.6546 [hep-ex].

[15] G. D'Ambrosio, G. F. Giudice, G. Isidori and A. Strumia, Nucl.Phys. B 645 (2002) 155, [hep$\mathrm{ph} / 0207036]$.

[16] D. M. Straub, arXiv:1012.3893 [hep-ph]; G. Isidori, F. Mescia, P. Paradisi, C. Smith and S. Trine, JHEP 0608, 064 (2006) [hep-ph/0604074].

[17] A. Crivellin, G. D’Ambrosio, T. Kitahara and U. Nierste, arXiv:1703.05786 [hep-ph].

[18] M. Endo, T. Kitahara, S. Mishima and K. Yamamoto, arXiv:1612.08839 [hep-ph].

[19] G. D'Ambrosio and D. N. Gao, JHEP 0010, 043 (2000) doi:10.1088/1126-6708/2000/10/043 [hep-ph/0010122]; L. Cappiello and G. D’Ambrosio, Phys. Rev. D 75, 094014 (2007) [arXiv:hep-ph/0702292]. 
[20] J. R. Batley et al. [NA48/2 Collaboration], Eur. Phys. J. C 68, 75 (2010) [arXiv:1004.0494 [hepex]].

[21] J. Bijnens, G. Colangelo, G. Ecker and J. Gasser, arXiv:hep-ph/9411311 and references there

[22] L. Cappiello, O. Cata, G. D’Ambrosio and D. -N. Gao, Eur. Phys. J. C 72, 1872 (2012) [arXiv:1112.5184 [hep-ph]].

[23] S. R. Gevorkyan and M. H. Misheva, Eur. Phys. J. C 74, no. 5, 2860 (2014) [arXiv:1403.1053 [hep-ph]].

[24] H. Pichl, Eur. Phys. J. C 20, 371 (2001) [hep-ph/0010284].

[25] Brigitte Bloch-Devaux On behalf of the NA48/2 collaboration "Study of the $K^{ \pm} \rightarrow \pi^{ \pm} \pi^{0} e^{+} e^{-}$ decay with NA48/2 @CERN": these proceedings.

[26] G. D'Ambrosio, G. Ecker, G. Isidori and J. Portoles, JHEP 9808, 004 (1998) doi:10.1088/11266708/1998/08/004 [hep-ph/9808289].

[27] A. Crivellin, G. D’Ambrosio, M. Hoferichter and L. C. Tunstall, Phys. Rev. D 93, no. 7, 074038 (2016) doi:10.1103/PhysRevD.93.074038 [arXiv:1601.00970 [hep-ph]].

[28] G. Isidori and R. Unterdorfer, JHEP 0401, 009 (2004) [hep-ph/0311084].

[29] G. Ecker and A. Pich, Nucl. Phys. B 366, 189 (1991);

[30] G. D’Ambrosio, D. Greynat and G. Vulvert, Eur. Phys. J. C 73, 2678 (2013) [arXiv:1309.5736 [hep-ph]]. 